\title{
Les Produits forestiers Turpin et l'essaimage: un modèle d'affaires intégré
}

\author{
Julie Bilodeau, Danielle Luc et Louis Jacques Filion ${ }^{1}$ \\ HEC-M ontréal ${ }^{2}$
}

Dans le domaine du sciage de bois de feuillus, Produits forestiers Turpin apparaît comme un véritable centre forestier. Au début de l'année 2001, cette entreprise comprenait une usine exploitant trois lignes de sciage, deux lignes de billons, un atelier de rabotage et six séchoirs. Trois autres entreprises, des essaimées de Produits forestiers Turpin installées dans son giron, s'occupent de l'exploitation forestière, du tronçonnage et de la fabrication de planchers de bois de wagons de chemin de fer. Générant un revenu annuel de plus de 30 millions de dollars, cette entreprise, qui a connu une croissance fulgurante, représente un des piliers économiques de la région de l'Outaouais. Propriétaire et gestionnaire principal, Germain Turpin se retrouve ainsi à la tête de la plus grande scierie de feuillus en Amérique du Nord. Son entreprise constitue d'ailleurs un des chefs de file de l'industrie.

\section{Son modèle d'affaires : l'essaimage}

Le succès impressionnant de Germain Turpin est en partie redevable à l'appui initial de son ex-employeur, Bruce Little. Ce dernier lui a permis d'acheter, à un prix moindre, la scierie en difficulté tout en lui fournissant une aide financière et technique non négligeable. Germain Turpin a remis sur pied cette entreprise déficitaire en un temps record. Cette association lui a d'ailleurs permis d'atteindre des sommets inespérés. S'appuyant sur son expérience personnelle, Germain Turpin croit aujourd'hui que cette forme de partenariat, soit l'essaimage, constitue une stratégie adéquate pour favoriser la croissance de son organisation. Durant les cinq dernières années, il a réalisé trois essaimages qui ont permis à l'entreprise de croître davantage et d'être plus concurrentielle. L'essaimage constitue donc aujourd'hui un modèle d'affaires intégré chez Produits forestiers Turpin. Il s'agit d'ailleurs d'un des plus beaux exemples d'entreprise essaimante au Québec. croissance fulgurante, représente un des piliers

e l'Outaouais. L'essaimage constitue dans la philosophie de l'entreprise.
Fruit de nombreuses expériences, le modèle d'essaimage créé par Germain Turpin se caractérise par plusieurs éléments qui présentent, selon lui, les conditions de succès d'une telle stratégie de croissance. L'essaimage doit générer des avantages pour les deux parties concernées. De plus, tous les entrepreneurs des entreprises essaimées sont choisis avec soin. Germain Turpin considère primordial qu'il y ait une bonne affinité entre lui et les entrepreneurs puisqu'il voit l'essaimage comme une association. En effet, une fois la nouvelle entreprise créée, il continue à maintenir des relations étroites avec les nouveaux dirigeants et à travailler conjointement avec eux. Il joue un rôle actif dans les décisions stratégiques sans toutefois s'occuper de la gestion quotidienne. La proximité géographique des essaimées de Produits forestiers Turpin facilite grandement le maintien de cette collaboration.

En outre, afin de maintenir la motivation et de reconnaître le travail accompli, Germain Turpin redistribue une partie importante des profits aux entrepreneurs essaimés. Comme ils possèdent entre 40 et $60 \%$ des parts de leur entreprise, les entrepreneurs sont des partenaires financiers majeurs. Ils exercent le pouvoir de direction de leur entreprise. Afin de faciliter le démarrage des entreprises essaimées, Germain Turpin fournit un soutien consultatif, technique et financier substantiel. Il a d'ailleurs conclu les mêmes ententes avec chaque entrepreneur. L'ensemble de ces entreprises est géré à 
partir d'une structure administrative commune, centralisée aux bureaux de Produits forestiers Turpin. Ce modèle particulièrement fructueux constitue en quelque sorte la « recette » d'essaimage de Germain Turpin, qui compte d'ailleurs l'appliquer lors de ses prochains essaimages.

\section{La création de Produits forestiers Turpin et la conception d'un modèle d'essaimage}

La création de Produits forestiers Turpin résulte à la fois d'une occasion d'affaires saisie par un entrepreneur qui croyait en son projet et d'un appui important de l'entreprise essaimante: Les Industries James Maclaren. Ce projet a permis de sauver l'usine de sciage de Thurso qui éprouvait, en 1991, de graves problèmes financiers. Cette expérience a d'ailleurs changé la vie de Germain Turpin.

Construite au début du $\mathrm{XX}^{\mathrm{e}}$ siècle par la société Singer pour produire le bois entrant dans la fabrication de ses machines à coudre, l'usine de sciage de bois de feuillus de Thurso constitue une des bases de l'économie et de l'histoire de cette petite localité de l'Outaouais. En effet, elle a créé de nombreux emplois durant plusieurs décennies. Propriété de Singer jusqu'en 1965, elle fut achetée par la multinationale Les Industries James Maclaren, laquelle sera ensuite affiliée au Groupe Noranda au début des années 1980. En 1991, une récession économique frappe l'Amérique du Nord. Le secteur des pâtes et papiers est durement touché et la demande de bois de sciage est à la baisse. Le $1^{\text {er }}$ mars 1991, la direction des Industries James Maclaren se voit obligée de fermer l'usine de sciage de Thurso. Les scénarios qui s'offrent sont soit la vente, soit la démolition de cette usine dont on ne sait plus que faire.

Directeur de l'usine à ce moment-là, Germain Turpin qualifie cette période de très difficile. En effet, à l'été 1991, quelques mois après la fermeture, l'avenir de l'usine n'était toujours pas connu. Inquiète, son équipe exerça beaucoup de pression sur lui afin de connaître l'issue. Face à cette pression de son entourage de travail, Germain Turpin décida de s'enquérir de son président, Bruce Little. Devant l'urgence de la situation, ce dernier décida d'organiser une rencontre avec tous les vice-présidents et directeurs afin de prendre une décision finale lors d'un comité de travail prévu pour le 15 octobre 1991. Il demanda à Germain Turpin de préparer une présentation suggérant des scénarios pour relancer l'usine.

Lorsque Germain Turpin se remémore cette importante réunion qui devait bouleverser sa vie, il se souvient de tout, autant dans le détail des mots prononcés que dans les émotions qu'il revit lorsqu'il en parle :

« J'étais assis à la droite de M. Little et je présentais nos différents scénarios possibles. Alors que nous étions en train de discuter des divers plans, à 10 h 50, M. Little se leva. Il annonça qu'il décidait de vendre. Il soutenait qu'il était possible de faire fonctionner l'usine de pâte à papier sans celle de sciage. Sous le choc, tout le monde fut estomaqué. Le silence fut immédiat. Plusieurs montrèrent leur déception. Quelques minutes après l'annonce, $\mathrm{M}$. Little se pencha vers moi et il me demanda si je désirais acheter l'usine. Je fus surpris d'une telle proposition. En rigolant, je lui répondis que cela dépendait du prix. J'ai donc suivi M. Little dans son bureau, accompagné de notre vice-président des finances. Quarante minutes plus tard, je suis ressorti avec une entente de principe d'achat entre les mains qui stipulait que M. Little me donnerait un coup de main pour réaliser ce projet. »

Après avoir pris un tournant tout à fait inattendu, cette réunion peut être considérée comme l'événement fondateur de Produits forestiers Turpin. Malgré le risque entourant le projet, Germain Turpin explique bien la nécessité de relever le défi qui se posait alors à lui :

«Je ne pouvais pas concevoir que mon usine allait être détruite. Si je n'acceptais pas sa proposition, M. Little, qui ne voulait pas vendre à un étranger, ne s'en préoccuperait plus et l'usine disparaîtrait. Une telle fin était pour moi inconcevable. Je trouvais que cela n'avait aucun sens. Je ne pouvais laisser tomber 115 employés. Juste à y penser, j'en avais des frissons dans le dos. Si je ne tentais pas ma chance, $j$ 'allais le regretter toute ma vie en me disant que j'aurais dû relancer l'usine. J'ai donc décidé de l'acheter. J'étais prêt à tout. »

Pour financer le lancement de son projet, Germain Turpin a éprouvé beaucoup de difficultés à se trouver des partenaires. Certaines institutions financières trouvaient le projet trop risqué,surtout en pleine période de récession. D'autres manifestaient un certain intérêt mais imposaient des conditions inacceptables. Le 30 janvier 1992, Germain Turpin signa finalement une entente de principe avec la Banque Nationale, mais l'annonce de la faillite de la scierie Davidson devait bientôt l'annuler. 
Actif dans le domaine depuis plus de trente ans et alors directeur de l'usine de sciage, Germain Turpin en connaît bien les rouages, de même que les raisons de sa non-rentabilité. Il a toutefois réfléchi aux solutions pour rendre cette opération rentable et profitable. Par exemple, prendre des décisions mensuelles et hebdomadaires plutôt qu'annuelles; réviser régulièrement les stratégies ou encore engager des contractuels pour des besoins ponctuels de spécialistes plutôt que de verser un montant fixe à la société mère pour n'avoir qu'occasionnellement recours à son personnel spécialisé.

Fort de cet avantage, malgré le grand défi rattaché à la recherche de financement, Germain Turpin réussit à démontrer le bien-fondé de son projet. Le 6 février 1992, il signa une entente avec Lucien Bergevin, vice-président de la Caisse centrale Desjardins, qui lui avait fait précédemment une offre. Ce dernier accepta de lui prêter les fonds nécessaires au redémarrage de la scierie, pour l'achat d'équipements et pour les réparations nécessaires à la bonne marche des opérations. La valeur de ce prêt et de la marge de crédit qui l'accompagne est d'un million de dollars: "Il n'était pas un expert forestier. Par contre, après avoir bien analysé mon plan de restructuration de l'exploitation, il a cru en moi et il a décidé de me faire confiance.»

Cette entente ne se concrétisa toutefois pas sans conditions. En effet, la Caisse centrale Desjardins demanda à la multinationale, soit l'entreprise essaimante, de s'engager à financer Germain Turpin pour l'achat de l'usine. Germain Turpin disposerait ainsi de cinq ans pour rembourser le coût de son achat. Les Industries James Maclaren devaient aussi s'engager à acheter la pâte et les sciures (copeaux) de la scierie peu importe les besoins. Finalement, elle devrait fournir de la vapeur et de l'électricité à un bon prix (légèrement au-dessus du prix coûtant) pour une période de cinq ans. Bruce Little s'engagea à respecter ces exigences. Convaincu de pouvoir rentabiliser son usine, Germain Turpin était prêt à risquer sa situation personnelle. Il hypothéqua l'ensemble de ses biens personnels et contracta en plus un cautionnement personnel d'un million de dollars.

Ces moments cruciaux demeurent profondément gravés dans sa mémoire. Germain Turpin se souvient clairement des événements qui ont marqué la naissance de sa nouvelle entreprise :

«J'ai créé Produits forestiers Turpin le 16 avril 1992 à 17 h 05 précises. J'ai acheté l'usine de sciage et l'usine de Doweloc, qui fabrique des planchers de bois pour les wagons de chemin de fer, à la multinationale Les Industries James Maclaren.»

Le 20 avril 1992, accompagné d'une équipe d'une vingtaine de personnes, Germain Turpin amorça les travaux de réparation de l'usine de sciage. Les opérations débutèrent le 11 mai 1992 avec un premier quart de travail. À ce moment, Produits forestiers Turpin comptait déjà 65 employés.

\section{Une croissance accélérée}

Bien qu'il ait eu confiance en son projet, Germain Turpin n'aurait jamais cru que Produits forestiers Turpin puisse croître aussi rapidement. En effet, au mois de juin 1992, l'ouragan Andrew frappa sauvagement la Floride. Détruisant nombre de maisons et bâtiments, son passage entraîna une forte demande de bois de sciage au cours de l'été qui suivit. Son usine dut par conséquent répondre à des besoins plus importants que prévus. Afin de pallier cette demande, Germain Turpin décida de mettre sur pied un deuxième quart de travail le 17 août 1992. Une catastrophe naturelle est venue favoriser la lancée de sa nouvelle organisation. À l'automne 1992, Produits forestiers Turpin réunissait déjà 140 employés.

En janvier 1993, le président de Produits forestiers Turpin, après avoir acheté l'usine de Doweloc et souhaitant obtenir de nouveaux contrats afin de la relancer, contacta un ami qui travaillait chez Canadien National. Rencontré deux ans auparavant dans le cadre d'une formation intensive sur la gestion des années 2000, cet ami le présenta au vice-président du CN. Après avoir signé un contrat avec ce dernier, Germain Turpin put relancer les activités de l'usine de Doweloc. Il embaucha par conséquent 25 employés. Le 16 avril 1993, un an après sa création, Produits forestiers Turpin regroupait 165 employés.

\section{Le 16 avril 1993, un an après sa création, Produits forestiers Turpin regroupait 165 employés. En 2000, huit ans après sa création, cette entreprise représente un succès et fait la fierté du village de Thurso.}

En 2000, huit ans après sa création, cette entreprise représente un succès et fait la fierté du village de Thurso. En effet, elle a connu une croissance impressionnante. 
Germain Turpin a gagné son pari. Son plan d'affaires s'est réalisé comme prévu. Germain Turpin a remboursé toutes les sommes empruntées à son entreprise essaimante et à la Caisse centrale Desjardins. Cette dernière se réjouit d'avoir investi dans un projet qui a entrainé une si importante création d'emplois. En 2000, Produits forestiers Turpin a d'ailleurs contracté d'autres emprunts envers cette institution pour financer ses derniers projets.

\section{La naissance d'un modèle émergent}

\section{Un contexte organisationnel qui favorise l'essaimage}

L'essaimage constitue une stratégie bien ancrée dans la philosophie de gestion de Germain Turpin. Il affirme d'ailleurs que cette formule lui permet de croître davantage et de manière efficace, assurant ainsi une meilleure productivité et une compétitivité soutenue. Étonnant au premier abord, le succès des essaimages réalisés par Germain Turpin s'explique en grande partie par un contexte bien particulier. D'un naturel confiant envers les personnes, Germain Turpin laisse beaucoup de latitude à ses employés. Il favorise l'engagement et la responsabilisation de tout son personnel en encourageant la coopération, l'initiative et la prise de risques.

Étonnant au premier abord, le succès des essaimages réalisés par Germain Turpin s'explique en grande partie par un contexte bien particulier. Ce dernier favorise

l'engagement et la responsabilisation de tout son personnel en encourageant la coopération, l'initiative et la prise de risques.

\section{L'histoire des quatre essaimages}

\section{Centre de tronçonnage Riopel :} une première tentative réussie

Le Centre de tronçonnage Riopel constitue le premier essaimage réalisé par Germain Turpin. Comme sa propre entreprise est le fruit d'une telle stratégie, il en connaît bien les rouages. Il en a même intégré le modèle dans ses façons d'être et de faire. Cette expérience s'est bien déroulée et les deux parties, Germain Turpin et Michel Riopel, en ressortent gagnantes. En l'an 2000, approchant de sa cinquième année d'existence, cette entreprise regroupait 26 employés et fonctionnait à pleine capacité. Son chiffre d'affaires a cru de $30 \%$ entre 1999 et 2000.
Concrétisé durant l'été 1996, le centre de tronçonnage constitue le résultat d'une occasion d'affaires saisie par les deux entrepreneurs. Ces derniers étaient convaincus de la réussite de leur projet, lancé à la suite de la fermeture d'un centre de tronçonnage par la multinationale Les Industries James Maclaren. En acceptant la proposition de l'un des opérateurs, Michel Riopel, de s'associer dans l'achat de cette entreprise, Germain Turpin s'est concentré davantage sur son activité principale, le sciage du bois. Une fois les ententes convenues entre les deux organisations et après l'installation de la tronçonneuse, les activités ont commencé, soit à la fin de septembre 1996.

Germain Turpin a fourni $60 \%$ de la mise de fonds nécessaire pour l'achat de l'équipement d'une valeur de 200000 \$, une aide technique ainsi que la possibilité d'utiliser la structure administrative de Produits forestiers Turpin (services de la comptabilité, des ressources humaines, téléphone, électricité, etc.) Il a ainsi apporté un précieux soutien à ce nouvel entrepreneur. La proximité des deux entreprises facilite d'ailleurs l'échange de services.

Avec, à toutes fins utiles, un seul client, soit Produits forestiers Turpin, le centre de tronçonnage s'occupe de la coupe des arbres en billes et billots ainsi que du transport vers l'usine de Thurso. Elle produit aussi de la pâte achetée ensuite par la multinationale Fraser (propriétaire des Industries James Maclaren depuis 2000). La pâte restante est aussi achetée par Produits forestiers Turpin, qui l'utilise pour fabriquer des copeaux qui sont ensuite vendus à Fraser. Ainsi, en plus de fournir le bois tronçonné à l'usine de sciage, Produits forestiers Turpin retire d'autres bénéfices en récupérant la pâte de bois et en limitant ainsi le gaspillage des ressources. Cette astucieuse pratique est d'ailleurs conforme au souci constant de protection des forêts de Germain Turpin et de son équipe.

Sous l'élan du premier essaimage, Germain Turpin décida de construire, en 1996, une autre usine de sciage à Maniwaki afin de répondre à la demande de plus en plus forte en ce domaine. Cette fois, il s'associa à Roger Riel (50\% de la propriété). Ayant employé plus de cent personnes, cette usine dut cependant être fermée trois ans plus tard parce qu'on n'arrivait pas à la rentabiliser. Expliquant cet échec par la difficulté de gérer à distance et par des erreurs de gestion, Germain Turpin affirme avoir appris beaucoup de cette expérience. Elle lui a 
permis de peaufiner son modèle d'essaimage. Ne pouvant être récupérée, cette usine a toutefois engendré des pertes financières importantes à Produits forestiers Turpin.

\section{Exploitation forestière Nations}

Satisfait de son association avec Michel Riopel, Germain Turpin décida de tenter un nouveau partenariat avec Yvan Riopel, le frère de son premier associé. Germain Turpin lui proposa de devenir son partenaire en créant une nouvelle entreprise spécialisée dans l'exploitation forestière. Avec les mêmes ententes administratives et le même support technique et consultatif que son frère avait reçus, Yvan Riopel fonda l'entreprise Exploitation forestière Nations en mai 1997. Il en détient $60 \%$ des actions.

Ayant lui aussi Produits forestiers Turpin pour seul client, Yvan Riopel s'occupe d'une partie de la coupe de bois en forêts ainsi que du transport jusqu'au centre de tronçonnage. Cette entreprise très profitable croît en moyenne de $10 \%$ par année depuis sa création. Satisfait, Germain Turpin ne regrette en rien cette association, car elle lui a permis d'économiser des sommes importantes en obtenant de meilleurs prix des contractants indépendants mandatés également pour la coupe de bois. En effet, en utilisant comme point de référence les coûts d'exploitation de l'essaimée, Germain Turpin détient un plus grand poids lors des négociations avec ses autres fournisseurs forestiers.

\section{Renyco}

Achetée au même moment que l'usine de sciage de Thurso lors de la création de Produits forestiers Turpin, l'usine est spécialisée dans la production de planchers de bois de wagons de chemin de fer, selon le procédé breveté Doweloc. Elle éprouva toutefois des difficultés financières au printemps 1997. Générant beaucoup de soucis, mais seulement $5 \%$ du chiffre d'affaires de Produits forestiers Turpin, Germain Turpin décida de fermer l'usine déficitaire en décembre 1997. Il reçut une proposition intéressante de son directeur d'usine, Louis Reny: ce dernier désirait acheter l'usine et la relancer.

Intéressé, le président de Produits forestiers Turpin accepta cette idée à condition que les activités soient réorientées vers le marché résidentiel. Le nouvel entrepreneur devait ainsi terminer la production entamée de planchers de bois de wagons pour ensuite transformer l'usine et ainsi pénétrer un nouveau marché. Propriétaire à $45 \%$, Louis
Reny fonda Renyco, en mai 1998, avec l'aide de Germain Turpin (45\% de la propriété) et Sylvain Boivin, directeur de production de Produits forestiers Turpin $(10 \%$ de la propriété).

Avec une aide similaire et des ententes semblables aux deux essaimages précédents, Louis Reny réussit lui aussi à redresser avec succès cette usine qui emploie aujourd'hui 30 employés. Toujours dans la production de planchers de bois de wagons, les résultats obtenus, la première année, furent de $50 \%$ supérieurs à ceux attendus. Ils génèrent des ventes de 2,5 millions de dollars. Client de Produits forestiers Turpin, Renyco vend ses produits principalement aux Américains (95\% des ventes). Désirant pénétrer le marché résidentiel, Renyco entama la construction d'une nouvelle usine à Thurso en février 2001. La nouvelle production, qui a débuté en juin 2001, a créé 75 nouveaux emplois ${ }^{3}$.

\section{Les conditions déterminantes}

Essaimé dans un premier temps et essaimant à trois reprises, Germain Turpin a tiré plusieurs leçons concernant la mise en œuvre d'une telle pratique. Considérant la formule gagnante, il en a défini les lignes de conduite. Appliquées à chaque nouvel essaimage, elles représentent, selon lui, les conditions de succès d'une telle stratégie de croissance.

Lors de la création d'une essaimée, selon ses dires, l'entrepreneur joue un rôle important. Il en influence beaucoup la réussite. Pilier de la nouvelle organisation, il doit être prêt à y investir temps et énergie. C'est pourquoi Germain Turpin insiste sur la nécessité d'avoir confiance en l'individu, autant dans ses capacités que dans ses compétences. Il suggère d'établir une relation d'affaires uniquement avec une personne détenant une bonne expérience dans le domaine. Il conseille aussi de connaître personnellement l'entrepreneur avant d'engager toute relation d'affaires sérieuse.

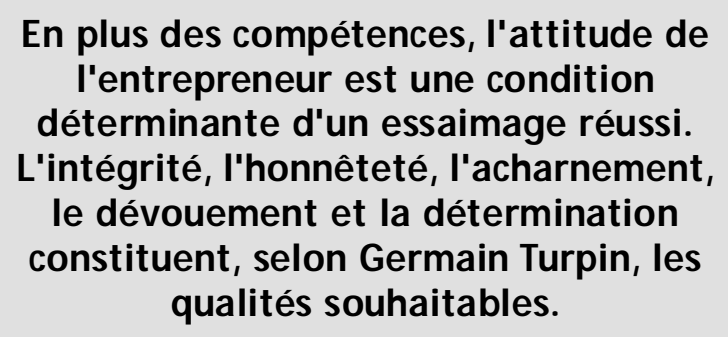

En plus des compétences, l'attitude de l'entrepreneur est une condition déterminante d'un essaimage réussi. L'intégrité, I'honnêteté, l'acharnement, le dévouement et la détermination constituent, selon Germain Turpin, les qualités souhaitables. 
En plus des compétences, l'attitude de l'entrepreneur est importante. L'intégrité, l'honnêteté, l'acharnement, le dévouement et la détermination constituent, selon Germain Turpin, les qualités souhaitables. L'entrepreneur doit vouloir réussir à tout prix et par conséquent, $\mathrm{y}$ investir les énergies et le temps requis tel qu'il l'a fait luimême lors du démarrage de Produits forestiers Turpin. En effet, durant les six premiers mois, il travaillait sept jours par semaine.

Si l'essaimante constitue un des partenaires d'affaires, comme c'est le cas chez Produits forestiers Turpin, une confiance mutuelle entre les deux entrepreneurs est essentielle pour la survie de la nouvelle association. Afin de faciliter cette relation, Germain Turpin conseille d'ailleurs de la clarifier dès le départ en officialisant les ententes par écrit. L'essaimante doit ainsi spécifier tous les détails et la durée de son engagement. Monsieur Turpin suggère d'éviter les ententes verbales afin de limiter les discussions inutiles, la perte de temps et les conflits éventuels. Puisque les besoins de l'essaimée évoluent au cours des mois, il conseille de réviser régulièrement les ententes et de les modifier, si nécessaire, afin que les deux parties soient satisfaites de leur association. Lorsqu'il évalue sa propre expérience, il est satisfait de ses relations avec ses essaimées :

«J'ai une bonne relation avec eux. Tout le monde est heureux. Ces trois entrepreneurs sont devenus de bons amis. Nous avons toujours réussi à nous entendre. Nos désaccords sont réglés à l'amiable. Nous avons une bonne communication.»

Afin de favoriser le dévouement de l'entrepreneur, Germain Turpin considère que ce dernier doit nécessairement être engagé sur le plan financier. En devenant un partenaire d'affaires important (à pourcentage élevé), il est récompensé pour les efforts investis. Cela encourage à travailler encore plus fort. Il est ainsi motivé à trouver des moyens de réduire les coûts. Cette formule a été utilisée dans les trois cas d'essaimage de Produits forestiers Turpin et a entraîné des résultats intéressants :

«Si elles n'avaient pas été partenaires financiers, les trois entreprises n'en seraient pas au stade actuel. Michel, Yvan et Louis n'auraient pas investi autant de temps et d'énergie. Ils travaillent fort mais ils en récoltent les bénéfices. »
Si l'essaimante constitue un des partenaires d'affaires, comme c'est le cas chez Produits forestiers Turpin, une confiance mutuelle entre les deux entrepreneurs est essentielle pour la survie de la nouvelle association.

\section{Les impacts de l'essaimage sur la croissance de Produits forestiers Turpin}

«Lorsque ton organisation a atteint un chiffre d'affaires de plus de 30 millions de dollars, il n'est pas possible de croître sans essaimage.» Voici la première affirmation de Germain Turpin sur les bienfaits de l'essaimage. Il s'agit selon lui d'une pratique qui procure la flexibilité requise pour être concurrentiel sur le marché mondial. Ce dernier ne regrette en rien ses associations. Ces trois entreprises satellites font qu'il est plus concurrentiel parce qu'en lui permettant de se concentrer davantage sur les activités de sciage, elles lui donnent accès à de meilleurs coûts d'approvisionnement. Ces unités d'affaires ne sont pas syndiquées et permettent ainsi à leurs dirigeants de sauver du temps en ce qui a trait à la gestion des ressources humaines. Étant donné leur engagement financier, les entrepreneurs des entreprises essaimées recherchent constamment des moyens d'améliorer leurs opérations. Les coûts de production sont inférieurs à ce qu'ils seraient si ces opérations étaient réalisées à l'intérieur d'une seule même grande entreprise. Et la qualité des produits et des services offerts est supérieure à ce qu'elle serait dans un contexte de grande entreprise.

Cette formule procure également un pouvoir de négociation accru auprès des autres fournisseurs. Exploitation forestière Nations constitue un bel exemple de cet avantage. En effet, ses coûts d'exploitation constituent un point de référence lors des négociations du prix d'achat du bois avec d'autres entrepreneurs indépendants. Produits forestiers Turpin peut ainsi obtenir de meilleurs prix et économiser plusieurs centaines de milliers de dollars par année dans l'exploitation forestière, diminuant ainsi les coûts et le prix de son produit final, le bois de sciage.

En restant éloigné de la gestion quotidienne des entreprises essaimées, Germain Turpin voit sa propre gestion simplifiée. En effet, en étant sur le terrain, les entrepreneurs peuvent s'attaquer plus rapidement aux divers problèmes. Finalement, l'essaimage a entraîné des répercussions intéressantes sur l'image de Produits forestiers Turpin. 
Par le biais des entreprises essaimées, Produits forestiers Turpin a favorisé la création de nombreux emplois dans une région où ils se font rares. Sensible à cette dimension, le gouvernement québécois et les institutions financières considèrent Produits forestiers Turpin comme une entreprise prospère et à l'avant-garde dans le secteur du sciage du bois de feuillus. Convaincu de la valeur supérieure de son modèle de stratégie de croissance, Germain Turpin encourage les entreprises à réaliser de l'essaimage. D'ailleurs, il prépare actuellement un quatrième essaimage, et ce ne sera sans doute pas le dernier.

\section{L'homme et son entreprise}

À l'âge de 45 ans, Germain Turpin, père de trois enfants, achète l'usine de sciage de Thurso, fermée depuis 14 mois et dont il était le directeur. Soutenu par son épouse, qui est aussi son adjointe administrative, et en y investissant toutes ses ressources et son énergie, il s'engage à relever le défi. Déterminé et confiant en son destin et en sa réussite, il s'accroche à ce rêve qui consiste à développer un centre forestier. Muni d'une excellente expérience et d'une expertise poussée en plus d'une intuition bien aiguisée, il réussit un projet ambitieux dans un contexte où les conditions n'étaient pas des plus favorables. Toujours en croissance, Produits forestiers Turpin représente, neuf ans plus tard, une belle réalisation.

Technicien forestier de formation de l'école de Duchesnay située près de Québec, Germain Turpin a travaillé pendant plus de 26 ans pour Les Industries James Maclaren. Il a débuté comme mesureur en 1965, il n'a cessé de progresser tout au long de sa carrière: il fut contremaître, chef mesureur, chef de service en 1980 , surintendant en 1984 et finalement, directeur de l'usine de sciage en 1987. Proche de Bruce Little, président, Germain Turpin est considéré comme le «fils corporatif». Son ancien patron a représenté et représente encore aux yeux de Germain Turpin un modèle à suivre.

Comme il a le souci de se perfectionner davantage en gestion, il entreprend différents cours dans ses temps libres, les soirs et les fins de semaine. Il suit des cours d'appoint en gestion des ressources humaines au cégep, puis il obtient un certificat en administration de l'Université du Québec.

Passionné par son travail, Germain Turpin est dévoué à son organisation. D'ailleurs, il travaille en moyenne
70 heures par semaine. Désirant poursuivre sa croissance, il innove à plusieurs niveaux. Il réfléchit constamment à de nouvelles approches pour améliorer les opérations et réduire les coûts. Il n'hésite pas à investir des sommes importantes dans l'amélioration de ses infrastructures.

Germain Turpin est aussi engagé socialement. En 1998, il a d'ailleurs occupé le poste de président de la campagne annuelle de Centraide Outaouais. Grâce à son dévouement, il a recueilli, avec l'aide de son équipe, plus de deux millions de dollars. Reconnu pour sa contribution sociale, il a été nommé la personnalité du mois d'avril 1999 par la Chambre de commerce et d'industrie de l'Outaouais.

\section{Profil actuel de Produits forestiers Turpin}

Spécialisée dans le sciage de bois de feuillus, Produits forestiers Turpin offre un vaste choix de bois de feuillus durs (érable, merisier, bouleau, frêle, chêne rouge, cerisier et tilleul). Ses produits sont utilisés pour la fabrication de parquets, de meubles, de boiseries et de palettes. L'entreprise vend $60 \%$ de sa production aux États-Unis. Le Québec et l'Ontario constituent ses autres marchés.

En vertu de deux contrats d'aménagement et d'approvisionnement forestiers avec le gouvernement québécois, qui lui donnent des droits de coupe pour une période de 25 ans, les terrains publics de la région de l'Outaouais représentent une de leurs principales sources de bois de feuillus, la matière première. Produits forestiers Turpin possède aussi des terrains privés de bois. Devant répondre à une grande demande, Produits forestiers Turpin doit aussi avoir recours aux producteurs indépendants, lesquels constituent leur dernière source d'approvisionnement.

Afin d'assurer sa croissance, Produits forestiers Turpin a acquis des scieries situées dans la région de Thurso. Par la propriété de terrains privés ou de droits de coupe sur des terrains publics, ces nouvelles usines assurent un plus grand bassin d'approvisionnement. En 1995, Produits forestiers Turpin a ainsi acheté l'usine de sciage de Papineauville regroupant 15 employés, et le 11 août 2000, l'usine de La Minerve, située dans la région de Lanaudière.

Hautement réputé dans l'industrie forestière, Germain Turpin souhaite que Produits forestiers Turpin devienne «le chef de file en transformation de feuillus nobles, par le développement de son potentiel humain et l'utilisation de technologies de pointe. L'entreprise vise à répondre aux exigences de la qualité de sa clientèle par l'amélioration 
continue des procédés de transformation en respectant le concept de foresterie durable et en regroupant un personnel motivét ${ }^{\star}$. D'ailleurs, la devise de l'entreprise stipule ce souci de la protection environnementale: «Nous jardinons la forêt de nos enfants.»

Soucieuse de se démarquer de la concurrence par la qualité de ses produits, Produits forestiers Turpin modernise régulièrement ses équipements de production. Depuis sa création, en 1992, elle a investi plus de 19 millions de dollars dans l'amélioration de ses infrastructures et opérations. En 1997, en collaboration avec la Banque de développement du Canada, Produits forestiers Turpin implante la norme ISO 9002. En 1998, l'entreprise réalise sa plus importante transformation. Elle annexe une deuxième usine de sciage à celle de Thurso, opération nécessitant un investissement de huit millions de dollars. Cette dernière se spécialise dans la coupe de billons, planches de bois de quatre à huit pieds, un format différent de celui trouvé habituellement sur le marché. Cette innovation permet à Produits forestiers Turpin de récupérer la pâte de papier inutilisée. Elle constitue d'ailleurs la première usine de bois courts au Québec.

\section{Les prochains défis}

\section{La relève : une préoccupation à moyen terme}

Germain Turpin songe déjà à planifier sa retraite, qu'il prévoit prendre dans environ cinq ans. Souhaitant que l'entreprise demeure au sein de la famille, il aimerait que son fils aîné, Garry, lui succède. Au début de la vingtaine, ce dernier étudie en génie électrique à l'École Polytechnique affiliée à l'Université de Montréal.

Germain Turpin envisage de vendre Produits forestiers Turpin si son fils ne lui succède pas. En l'occurrence, les frères Riopel se départiraient également de leur organisation. Appréciant leur relation avec Germain Turpin, ces derniers ne désirent pas amorcer une nouvelle association avec un inconnu.

\section{La poursuite de la croissance}

En plus de préparer un quatrième essaimage, Germain Turpin souhaite investir davantage dans les infrastructures au cours des prochaines années. Il prévoit acheter de nouveaux séchoirs d'une valeur d'un million de dollars et une bouilloire de 1,5 million de dollars pour produire de la vapeur. Il veut aussi améliorer l'usine de billots en y ajoutant dix employés, ce qui nécessitera également un investissement d'un million de dollars.

Finalement, il souhaite acheter des territoires privés de bois. Enjeu majeur des prochaines années au Québec, l'approvisionnement représente une des principales préoccupations du président. En effet, puisque le nombre de fabricants de planchers de bois augmente et que le bois de feuillus diminue, ceux qui ne possèdent pas de terrains privés et de droits de coupe trouveront les temps difficiles. La concurrence s'est également étoffée depuis trois ans. En effet, avec la mondialisation, les pays européens deviennent des concurrents importants, surtout que certains d'entre eux, tels la Russie et la Pologne, possèdent des forêts qui n'ont jamais été exploitées. Produits forestiers Turpin doit ainsi faire face à ces nouveaux joueurs et continuer à se distinguer par ses innovations, la qualité de ses produits et des prix compétitifs.

\section{Notes et références}

1 La rédaction de ce cas a été faite dans le cadre d'un projet de la Chaire d'entrepreneurship Maclean Hunter sur l'essaimage d'entreprises. Les partenaires financiers sont la Fondation de l'entrepreneurship, le ministère de l'Industrie et du Commerce, Développement économique Canada et Accès Entrepreneur Plus.

2 Ce cas est reproduit avec l'autorisation du Centre de cas de l'École des Hautes Études Commerciales de Montréal.

3 Dans le cadre du même projet, un cas a également été rédigé explicitant la création de Renyco.

4 Tel que cité sur le site Web de l'entreprise: www.pfturpin.com 